\title{
Towards Understanding the Origins of Quantum Indeterminism and Non-Local Quantum Jumps
}

\author{
M. Sadiq1, Fahad M. Alharbi' \\ ${ }^{1}$ Department of Physics, University of Tabuk, Tabuk, Saudi Arabia \\ ${ }^{2}$ Department of Mathematics, University of Tabuk, Tabuk, Saudi Arabia \\ Email: ms.khan@ut.edu.sa, falharbi@ut.edu.sa
}

How to cite this paper: Sadiq, M. and Alharbi, F.M. (2018) Towards Understanding the Origins of Quantum Indeterminism and Non-Local Quantum Jumps. Journal of Applied Mathematics and Physics, 6, 2468-2474.

https://doi.org/10.4236/jamp.2018.612208

Received: October 22, 2018

Accepted: December 4, 2018

Published: December 7, 2018

Copyright $\odot 2018$ by authors and Scientific Research Publishing Inc. This work is licensed under the Creative Commons Attribution International License (CC BY 4.0).

http://creativecommons.org/licenses/by/4.0/

\begin{abstract}
By Invoking symmetry principle, we present a self-consistent interpretation of the existing quantum theory which explains why our world is fundamentally indeterministic and that why non-local quantum jumps occur. Symmetry principle dictates that the concept of probability is more fundamental than the notion of the wave function in that the former can be derived directly from symmetries rather than have to be assumed as an additional axiom. It is argued that the notion of quantum probability and that of the wavefunction are intimately connected.
\end{abstract}

\section{Keywords}

Bohr-Einstein Debate, Copenhagen Interpretation, Indeterminism, Leibniz-Clarke Dialogue, Quantum Probability, Space and Time

\section{Introduction}

Quantum theory originated in the early $20^{\text {th }}$ century with the inability of classical physics to account for the observed spectral distribution of the black body radiation and the new experimental data related to the electronic structure of atoms and molecules. Through the works of Max Planck, Albert Einstein and Arthur Compton light began to be conceived as consisting of discrete packets (quanta) of energy, now known as photons. In 1913 the mathematical model of Neils Bohr for the hydrogen atom came, subsequently improved by the work of Arnold Sommerfeld. The next revolutionary idea that came in 1924 was de Broglie's hypothesis on the possibility of an electron and the like, having wave-like properties, and its subsequent verification by the seminal electron diffraction 
experiments by Davison and Germer in 1927. This provided impetus for the development of the elegant quantum mechanics. Influenced by Werner Heisenberg and many other giants, including Paul Dirac, Max Born, Erwin Schrödinger, Wolfgang Pauli and John von Neumann, to name only a few, the basic mathematical structure that we have today of quantum mechanics was formed. Max Jammer's classic book [1] dwells at length on the chronological account of the development of quantum formalism. The theory so developed consists of a complete and logically consistent framework of mathematical deductions that could be applied to any quantum physical system and is beyond doubt one of the major advances in the history of science. Since its beginning, quantum theory has never been found to be contradicted by any microscopic phenomenon. The spectacular advances in physics, chemistry, biology, electronics-and essentially every other science-could not have occurred without the wonderful tools made possible by the deep knowledge of the micro-structure that quantum mechanics has offered us. With its elegant mathematical structure, firstly, quantum mechanics solved with immense success mysteries ranging from macroscopic superconductivity to the microscopic theory of elementary particles. Secondly, this compelled physicists to revise drastically the pre-existing ideas regarding reality and to reshape the concepts of cause and effects when dealing with matter at the microscopic level.

Despite such profound success, however, quantum mechanics has been notoriously confusing when it comes to its interpretation. Since the early development of quantum mechanics, the concept of measurement or the collapse of the quantum wave function has been at the root of the controversy that found concrete expression in the historical Bohr-Einstein debates [2]. There emerged many interpretations of quantum mechanics differing over which physical processes are to be considered measurements; can the measurement process be understood in deterministic terms; what actually causes collapse of the wave function; which elements of quantum theory can be called as real; what is the role of a conscious observer in the measurement process, and other matters. Among the prominent interpretations are, for instance, the hidden variable theories (an example of which is the de Broglie-Bohm pilot wave theory [3] [4], the relative state formulation or the many worlds interpretation of quantum mechanics due to Haugh Everett [5] [6], the consistent histories interpretation [7], the ensemble or statistical interpretation [8], etc. We do not, however, intend to evaluate these various views regarding the foundations of quantum mechanics; in fact, a huge amount of work have been devoted to these topics but the puzzles that quantum theory generated persist to this day [9] [10]. One naturally wonders how it is possible that such an extraordinarily successful theory may lead to such dubious issues and so many contending interpretations.

Lucien Hardy [11] showed elaborately that quantum theory can follow from five very reasonable axioms which, being related to the classical probability theory, might well have been posited prior to any empirical data which became 
available at the beginning of the $20^{\text {th }}$ century leading ultimately to the foundations of quantum mechanics. One would however wonder to understand why a physical theory should be probabilistic at the fundamental level.

Symmetries have long been realized to play a fundamental role in quantum mechanics. The very basic algebraic structure and conservation laws in quantum mechanics follow from symmetry transformations on the wave function such as displacements and rotations through angles in space. But we argue that symmetries tell us more than what we think of them in the conventional account of the theory. In this paper, we wish to invoke symmetry rules to explain why there is indeterminism in our world in the first place. This in turn automatically explains why non-local quantum jumps are deeply rooted in the theory. Symmetry principle tells us that a quantum jump or collapse of the wave function is by its very nature instantaneous and that we cannot explain it in causal terms. Here we do not intend in the least to depart from the standard formulation of quantum mechanics but will try to make it clear that the role of symmetries in quantum mechanics is far more revealing than what is conventionally recognized. We will argue that in contrast to the traditional view the concept of probability is the prime ingredient of quantum mechanics that need not be imposed from outside as to give meaning to the wave function-it is rather the wave function which can be introduced into the picture as a mathematical tool to describe the intrinsic indeterminism resulting from symmetry.

We begin our discussion with a brief discussion of the measurement problem in Section 2. In Section 3, we present our view about the role of symmetries in understanding the emergence of indeterminism and non-locality. In Section 4, we outline the main conclusions which can be derived from this paper.

\section{The Measurement Problem}

Starting with John von Neumann [12] the orthodox school (also commonly known as the Copenhagen School of thought for historical reasons), led by Neil Bohr, holds that a wavefunction (the quantum state), represented by a state vector $\psi$ in a Hilbert space, evolves with time according to the deterministic Schrödinger equation when the quantum stateis not observed, and is in general given by a coherent superposition of various experimental results, i.e., $\psi=\sum c_{i} \phi_{i}$. Here $\phi_{j}$ 's represent the possible experimental results (eigenstates of an operator representing a dynamical variable that is being measured), and $c_{j}$ 's (in general complex) are the probability amplitudes for the various outcomes. The quantity $\left|c_{j}\right|^{2}$ is postulated as representing the probability of finding the quantum system in the state $\phi_{j}$. Moreover, since an immediately repeated measurement yields the same result, we are to assume that the act of measurement collapses the wavefunction $\psi$, as we say; in an instant the quantum system jumps into a definite state $\phi_{j}$ and all other possibilities simply cease to exist. For example, a particle's existence is spread out over the entire space and appears only in a localized region of space when one measures its position-the probability of finding the particle at all other points drops to zero instantly. This instantaneous col- 
lapse (reduction) of the wavefunction, also referred to as non-locality ${ }^{1}$, proved a source of conceptual difficulties which has come to be known as the measurement problem. The difficulties come about in the interpretation of the mechanism by which a definite state is instantly singled out from amongst all possible outcomes. In this regard the orthodox school holds that the act of measurement is strictly outside the explanatory reach of quantum mechanics and that it requires a separate axiom of measurement in addition to the formalism of quantum mechanics.

\section{Emergence of Indeterminism from Symmetry}

In a celebrated correspondence with Clarke, a disciple of Newton, Leibniz denied the independent existence of space and time [13] [14]. Leibniz described space as a relational notion that existed only as a relation between objects that had no independent existence apart from those objects; motions existed only as relations between those objects. Leibniz critiqued Newton's idea of the absolute space in which all points were exactly identical and relative to which all motions took place, by claiming that such a situation would have presented God with an impossible decision, i.e., where precisely to put the contents of the universe; why here rather than there? He argued that even God must have enough reason for all His acts; the impossibility of finding any such reason for any placement demonstrated that the notion of an absolute space could not be correct.

Yet one could offer a radically different description of the same kind of a problem which probably would have been far direr than what Leibniz might have thought, and which, in this author's view, also contained the seeds for a non-deterministic mechanics. To proceed, we assume that a continuous (smoothed-out) Newtonian space pre-exists out there-this is what both classical physics and the contemporary quantum theory rest on. In order to make the argument more explicit, we restrict our attention to a simplistic example of a one-dimensional circular space (that could be of any size with no preferable point-symmetry). Now, one is asked to put a point particle on the circle somehow. The word "somehow" is used deliberately for it indicates the real trouble-as pointed out by Leibniz; since all the points in space are indistinguishable, we can by no causal reasoning explain the mechanism through which this placement would take place. Here, suddenly, all the rules of local determinism which we are familiar with no longer hold. For a point particle to reside in such a naive place, where there is no preferable location, we will need a drastic revision of our fundamental concepts-a new kind of physical laws must be formulated in place of the traditional laws of classical mechanics. The only plausible way to accommodate the current theoretical problem is to postulate the following utterly unusual rules which have no classical counterparts:

First, "since all points on the circle are equivalent, each point has to share with equal 'weight' the existence of the 'whole' particle". A classical determinist would hardly be prepared to allow for this hypothesis because it abandons any notion

${ }^{1}$ That is, physical distances, and so the limitation of the speed of light, don't seem to exist. 
that a particle could have a well-defined position. This fuzzy non-classical behavior that the particle exhibits, i.e., having existence at all points on the circle at the same time, is what one might call as potential existence-a term which in its essence is no different from Heisenberg's "potentia" [15]. We may express it as probabilities of experimental outcomes and must therefore be distinguished from the mere existence in space as for classical objects. One should, however, recognize that there is a strong difference between the concept of (quantum) probability introduced here and the one what it is in classical physics.

The second, and probably more inexplicable, postulate is that, "when 'projected' into this space, the particle's potential existence has to spread over the entire space instantly". Note that this postulate, expressing non-locality of the theory, is certainly tied with the first one because otherwise the first postulate would be violated for the length of time, whatever, the particle takes to fill the available space with its potential existence. Remarkably, this instantaneous spread is the essence of discontinuous quantum jumps (i.e., the collapse phenomenon in which the particle picks up one observable value out of many) as will become even clearer when we formulate these postulates mathematically. Notable in all this approach is that the concept of probability and that of the discontinuous quantum jumps are not the concepts to be endowed arbitrarily into the theory (as is done in the conventional approach) but rather are necessitated by the symmetry rules and are mutually consistent. It is thus the symmetry rule that dictates us to adapt ourselves to the counter-intuitive notions as potential existence and non-local abrupt jumps, and we cannot make this weirdness go away by explaining how it works. It is therefore not wise to search for any causal explanation of the processes that are intrinsically probabilistic and non-local.

Let us now attempt to find out an appropriate mathematical formulation that would fit the description of this manifestly probabilistic realm. First of all note that the probability density on the circle should be constant and positive definite. Merely a positive definite constant $|A|^{2}$ (where $A$ may be a complex constant in general) would not do because the probability density should as well be expressed by a function of the angular variable $\varphi$ and of any possible "uniform circular flow" of the probability density. Therefore, we choose a function $|A f(m \varphi)|^{2}$ to represent the constant and positive definite probability density. Now, in order for $|A|^{2}$ to represent the constant probability density the right choice (apart from an overall phase factor) for the function $f(m \varphi)$ would be $A \exp [\operatorname{im} \varphi] . A$ must be chosen such that the total probability $\int|A \exp [\operatorname{im} \varphi]|^{2} \mathrm{~d} \varphi$ is unity. So, it naturally turns out that a point particle on a circle can be represented by a complex stationary circular wave $\psi(\varphi)=A \exp [\operatorname{im} \varphi]$. Demanding this function to be single-valued, $m$ should be restricted to the values 0 , $\pm 1, \pm 2, \ldots$ One of these values will be picked up by the particle while it interacts instantly with the circular space. Furthermore, note that for a large radius this circular wave function should reduce to a plane wave ${ }^{2}$. Thus, the reasoning pre${ }^{2}$ It is worth noting that identifying $m$ with the angular momentum it is straight forward to show that for a large circle the circular wave reduces to a plane wave with a single value of linear momentum. 
sented here naturally leads to the de Broglie's hypothesis as well as Bohr's discrete stationary states.

It is worth noting that the symmetry rules lead to the conclusion that the wave function and its probabilistic interpretation are intimately connected and, therefore, Born's probability hypothesis [16] no longer seems to be an ad hoc one. Thus, once it is conceived that there is a wave function associated with a particle, the usual mathematical structure of the quantum theory can be recovered.

\section{Conclusion}

We noted that symmetries are at the root of the indeterminism and non-local quantum jumps at the microscopic level. Quantum probability seems to be the primary ingredient of theory. Furthermore, the notions of probability and the wave function are interwoven together within the theory and do not require any axiomatic hypothesis from outside the theory.

\section{Acknowledgements}

The authors are indebted to the University of Tabuk for its continuous support.

\section{Conflicts of Interest}

The authors declare no conflicts of interest regarding the publication of this paper.

\section{References}

[1] Jammer, M. (1966) The Conceptual Development of Quantum Mechanics. McGraw Hill, New York.

[2] Whitaker, A. (1996) Einstein, Bohr and the Quantum Dilemma. University of Cambridge, Cambridge.

[3] Bohm, D.J. and Hiley, B.J. (1982) The de Broglie Pilot Wave Theory and the Further Development of New Insights Arising out of It. Foundations of Physics, 12, 1001-1006. https://doi.org/10.1007/BF01889273

[4] Bell, J.S. (1992) Six Possible Worlds of Quantum Mechanics. Foundations of Physics, 22, 1201-1215. https://doi.org/10.1007/BF01889711

[5] Everett, H. (1957) "Relative State" Formulation of Quantum Mechanics. Reviews of Modern Physics, 29, 454. https://doi.org/10.1103/RevModPhys.29.454

[6] Barrett, J.A. (1999) The Quantum Mechanics of Minds and Worlds. Oxford University, Oxford.

[7] Griffiths, R.B. (1993) Consistent Interpretation of Quantum Mechanics Using Quantum Trajectories. Physical Review Letters, 70, Article ID: 2201.

[8] Ballentine, L.E. (1970) The Statistical Interpretation of Quantum Mechanics. Reviews of Modern Physics, 42, 358. https://doi.org/10.1103/RevModPhys.42.358

[9] Bell, J.S. (1987) Speakable and Unspeakable in Quantum Mechanics. Cambridge University, Cambridge.

[10] Cushing, J.T. (1994) Quantum Mechanics, Historical Contingency and the Copenhagen Hegemony. The University of Chicago, Chicago. 
[11] Hardy, L. (2001) Quantum Theory from Five Reasonable Axioms. arXiv:quant$\mathrm{ph} / 0101012$.

[12] Neumann Von, J. (1932) Mathematische Grundlagen der Quantenmechanik [Mathematical Foundations of Quantum Mechanics]. Springer-Verlog, Princeton University Press, Berlin.

[13] Alexander, H.G. (1956) The Leibniz-Clarke Correspondence. University of Manchester, Manchester.

[14] Barbour, J. (1982) Relational Concepts of Space and Time. The British Journal for the Philosophy of Science, 33, 251-274.

[15] Heisenberg, W. (1958) Physics and Philosophy, the Revolution in Modern Science Harper, New York.

[16] Born, M. (1926) Quantenmechanik der stoßvorgänge. Zeitschrift für Physik, 38, 803-827. 